\title{
Tweaking the Beukers Integrals In Search of More Miraculous Irrationality Proofs À La Apéry
}

\author{
Robert DOUGHERTY-BLISS, Christoph KOUTSCHAN, and Doron ZEILBERGER
}

In honor of our irrational guru Wadim Zudilin, on his $\lfloor 50 \zeta(5)\rfloor$-th birthday

\section{[Actual] Historical Introduction: How Beukers' Proofs Were ACTUALLY found}

\section{Hilbert's 0-th problem}

Before David Hilbert $[\mathrm{H}]$ stated his famous 23 problems, he mentioned two problems that he probably believed to be yet much harder, and indeed, are still wide open today. One of them was to prove that there are infinitely many prime numbers of the form $2^{n}+1$, and the other one was to prove that the Euler-Mascheroni constant is irrational.

Two paragraphs later he stated his optimistic belief that "in mathematics there is no ignorabimus."

As we all know, he was proven wrong by Gödel and Turing in general, but even for such concrete problems, like the irrationality of a specific, natural, constant, like the Euler-Mascheroni constant (that may be defined in terms of the definite integral $\quad-\int_{0}^{\infty} e^{-x} \log x$ ), that is most probably decidable in the logical sense, (i.e. there probably exists a (rigorous) proof), we lowly humans did not yet find it, (and may never will!).

While the Euler-Mascheroni constant (and any other, natural, explicitly-defined, constant that is not obviously rational) is surely irrational, in the everyday sense of the word sure (like death and taxes), giving a proof, in the mathematical sense of 'proof' is a different matter. While $e$ was proved irrational a long time ago (trivial exercise), and $\pi$ was proved irrational by Lambert around 1750, we have no clue how to prove that $e+\pi$ is irrational. Ditto for $e \cdot \pi$. Exercise: Prove that at least one of them is irrational.

\section{Apéry's Miracle}

As Lindemann first proved in 1882, the number $\pi$ is more than just irrational, it is transcendental, hence it follows that $\zeta(n)$ is irrational for all even arguments, since Euler proved that $\zeta(2 n)$ is a multiple of $\pi^{2 n}$ by a rational number. But proving that $\zeta(3), \zeta(5), \ldots$ are irrational remained wide open.

Since such problems are so hard, it was breaking news, back in 1978, when 64-years-old Roger Apéry announced and sketched a proof that $\zeta(3):=\sum_{n=1}^{\infty} \frac{1}{n^{3}}$ is irrational. This was beautifully narrated in a classic expository paper by Alf van der Poorten $[\mathrm{vdP}]$, aided by details filled-in by Henri Cohen and Don Zagier. While beautiful in our eyes, most people found the proof ad-hoc and too complicated, and they did not like the heavy reliance on recurrence relations.

To those people, who found Apéry's original proof too magical, ad-hoc, and computational, another proof, by a 24-year-old $\mathrm{PhD}$ student by the name of Frits Beukers [B] was a breath of fresh air. It 
was a marvelous gem in human-generated mathematics, and could be easily followed by a first-year student, using partial fractions and very easy estimates of a certain triple integral, namely

$$
\int_{0}^{1} \int_{0}^{1} \int_{0}^{1} \frac{(x(1-x) y(1-y) z(1-z))^{n}}{(1-z+x y z)^{n+1}} d x d y d z
$$

The general approach of Apéry of finding concrete sequences of integers $a_{n}, b_{n}$ such that

$$
\left|\zeta(3)-\frac{a_{n}}{b_{n}}\right|<\frac{C O N S T}{b_{n}^{1+\delta}}
$$

(see below) for a positive $\delta$ was still followed, but the details were much more palatable and elegant to the average mathematician in the street.

As a warmup, Beukers, like Apéry before him, gave a new proof of the already proved fact that $\zeta(2)=\frac{\pi^{2}}{6}$ is irrational, using the double integral

$$
\int_{0}^{1} \int_{0}^{1} \frac{(x(1-x) y(1-y))^{n}}{(1-x y)^{n+1}} d x d y
$$

Ironically, we will follow Beukers' lead, but heavily using recurrence relations, that will be the engine of our approach. Thus we will abandon the original raison d'être of Beukers' proof of getting rid of recurrences, and bring them back with a vengeance.

\section{[Alternative World] Historical Introduction: How Beukers's Proofs Could (and Should!) have been Discovered}

Once upon a time, there was a precocious teenager, who was also a computer whiz, let's call him/her/it/they Alex. Alex just got a new laptop that had Maple, as a birthday present.

Alex typed, for no particular reason,

$\operatorname{int}(\operatorname{int}(1 /(1-x * y), x=0 \ldots 1), y=0 \ldots 1)$;

and immediately got the answer: $\frac{\pi^{2}}{6}$. Then Alex was wondering about the sequence

$$
I(n):=\int_{0}^{1} \int_{0}^{1} \frac{(x(1-x) y(1-y))^{n}}{(1-x y)^{n+1}} d x d y .
$$

(why not, isn't it a natural thing to try out for a curious teenager?), and typed

$\mathrm{I} 1:=\mathrm{n}->\operatorname{int}(\operatorname{int}(1 /(1-\mathrm{x} * \mathrm{y}) *(\mathrm{x} *(1-\mathrm{x}) * \mathrm{y} *(1-\mathrm{y}) /(1-\mathrm{x} * \mathrm{y})) * * \mathrm{n}, \mathrm{x}=0 \ldots 1), \mathrm{y}=0 \ldots 1)$;

(I is reserved in Maple for $\sqrt{-1}$, so Alex needed to use I1),

and looked at the first ten values by typing: 
$\mathrm{L}:=[\operatorname{seq}(\mathrm{I} 1(\mathrm{i}), \mathrm{i}=1 \ldots 10)] ;$,

getting after a few seconds

$$
\begin{gathered}
{\left[5-\frac{\pi^{2}}{2},-\frac{125}{4}+\frac{19 \pi^{2}}{6}, \frac{8705}{36}-\frac{49 \pi^{2}}{2},-\frac{32925}{16}+\frac{417 \pi^{2}}{2},\right.} \\
\frac{13327519}{720}-\frac{3751 \pi^{2}}{2},-\frac{124308457}{720}+\frac{104959 \pi^{2}}{6}, \\
\frac{19427741063}{11760}-\frac{334769 \pi^{2}}{2},-\frac{2273486234953}{141120}+\frac{9793891 \pi^{2}}{6}, \\
\left.\frac{202482451324891}{1270080}-\frac{32306251 \pi^{2}}{2},-\frac{2758128511985}{1728}+\frac{323445423 \pi^{2}}{2}\right] .
\end{gathered}
$$

Alex immediately noticed that, at least for $n \leq 10$,

$$
I(n)=a_{n}-b_{n} \frac{\pi^{2}}{6}
$$

for some integers $b_{n}$ and some rational numbers $a_{n}$. By taking evalf(L), Alex also noticed that $I(n)$ get smaller and smaller. Knowing that Maple could not be trusted with floating point calculations (unless you change the value of Digits from its default, to something higher, say, in this case Digits:=30), that they get smaller and smaller. Typing 'evalf $(\mathrm{L}, 30)$;', Alex got:

[0.06519779945532069058275450006, 0.0037472701163022929758881663,

0.000247728866269394110526059, 0.00001762713127202699137347,

$0.0000013124634659314676853,0.000000100776323486001254$,

0.00000000791212964371946, 0.0000000006317437711206,

$$
\left.5.1111100706 \times 10^{-11}, 4.17922459 \times 10^{-12}\right] .
$$

Alex realized that $I(n)$ seems to go to zero fairly fast, and since $I(10) / I(9)$ and $I(9) / I(8)$ were pretty close, Alex conjectured that the limit of $I(n) / I(n-1)$ tends to a certain constant. But ten data points do not suffice!

When Alex tried to find the first 2000 terms, Maple got slower and slower. Then Alex asked Alexa, the famous robot,

Alexa: how do I compute many terms of the sequence $I(n)$ given by that double-integral?

and Alexa replied:

Go to Doron Zeilberger's web-site and download the amazing program

https://sites.math.rutgers.edu/〜zeilberg/tokhniot/MultiAlmkvistZeilberger.txt , 
that accompanied the article [ApaZ]. Typing

$\operatorname{MAZ}(1,1 /(1-\mathrm{x} * \mathrm{y}), \mathrm{x} *(1-\mathrm{x}) * \mathrm{y} *(1-\mathrm{y}) /(1-\mathrm{x} * \mathrm{y}),[\mathrm{x}, \mathrm{y}], \mathrm{n}, \mathrm{N},\{\})[1]$

immediately gave a recurrence satisfied by $I(n)$

$$
I(n)=-\frac{\left(11 n^{2}-11 n+3\right)}{n^{2}} \cdot I(n-1)+\frac{(n-1)^{2}}{n^{2}} \cdot I(n-2)
$$

Using this recurrence, Alex easily computed the first 2000 terms, using the following Maple one-liner (calling the sequence defined by the recurrence I2(n)):

I2: $=\operatorname{proc}(\mathrm{n})$ option remember: if $\mathrm{n}=0$ then $\mathrm{Pi} * * 2 / 6$ elif $\mathrm{n}=1$ then $5-\mathrm{Pi} * * 2 / 2$ else $-(11 * \mathrm{n} * * 2-$ $11 * n+3) / n * * 2 * I 2(n-1)+(n-1) * * 2 / n * * 2 * I 2(n-2): f i:$ end:

and found out that indeed $I(n) / I(n-1)$ tends to a limit, about 0.09016994 . Writing

$$
I(n)=a_{n}-b_{n} \frac{\pi^{2}}{6}
$$

and realizing that $I(n)$ is small, Alex found terrific rational approximations to $\frac{\pi^{2}}{6}, a_{n} / b_{n}$, that after clearing denominators can be written as $a_{n}^{\prime} / b_{n}^{\prime}$ where now both numerator $a_{n}^{\prime}$ and denominator $b_{n}^{\prime}$ are integers.

$$
\frac{\pi^{2}}{6} \approx \frac{a_{n}^{\prime}}{b_{n}^{\prime}}
$$

Alex also noticed that for all $n$ up to 2000 , for some constant $C$,

$$
\left|\frac{\pi^{2}}{6}-\frac{a_{n}^{\prime}}{b_{n}^{\prime}}\right| \leq \frac{C}{\left(b_{n}^{\prime}\right)^{1+\delta}},
$$

where $\delta$ is roughly 0.09215925467 . Then Alex concluded that this proves that $\frac{\pi^{2}}{6}$ is irrational, since if it were rational the left side would have been $\geq \frac{C_{1}}{b_{n}^{\prime}}$, for some constant $C_{1}$. Of course, some details would still need to be filled-in, but that was not too hard.

\section{The General Strategy}

Let's follow Alex's lead. (Of course our fictional Alex owes a lot to the real Beukers and also to Alladi and Robinson $[\mathrm{AR}])$.

Start with a constant, let's call it $C$, given by an explicit integral

$$
\int_{0}^{1} K(x) d x
$$

for some integrand $K(x)$, or, more generally, a $d$-dimensional integral

$$
\int_{0}^{1} \ldots \int_{0}^{1} K\left(x_{1}, \ldots, x_{k}\right) d x_{1} \ldots d x_{k}
$$


Our goal in life is to prove that $C$ is irrational. Of course $C$ may turn out to be rational (that happens!), or more likely, an algebraic number, or expressible in terms of a logarithm of an algebraic number, for which, there already exist irrationality proofs (albeit not always effective ones). But who knows? Maybe this constant has never been proved irrational, and if it will happen to be famous (e.g. Catalan's constant, or $\zeta(5)$, or the Euler-Mascheroni constant mentioned above), we will be famous too. But even if it is a nameless constant, it is still extremely interesting, if it is the first irrationality proof, since these proofs are so hard, witness that, in spite of great efforts by experts like Wadim Zudilin, the proofs of these are still wide open.

In this article we will present numerous candidates. Our proofs of irrationality are modulo a 'divisibility lemma' (see below), that we are sure that someone like Wadim Zudilin, to whom this paper is dedicated, can fill-in. Our only doubts are whether these constants are not already proved to be irrational because they happen to be algebraic (probably not, since Maple was unable to identify them), or more complicated numbers (like logarithms of algebraic numbers). Recall that Maple's identify can't (yet) identify everything that God can.

Following Beukers and Alladi-Robinson, we introduce a sequence of integrals, parameterized by a non-negative integer $n$

$$
I(n)=\int_{0}^{1} K(x)(x(1-x) K(x))^{n} d x,
$$

and analogously for multiple integrals, or more generally

$$
I(n)=\int_{0}^{1} K(x)(x(1-x) S(x))^{n} d x
$$

for another function $S(x)$. Of course $I(0)=C$, our constant that we want to prove irrational.

It so happens that for a wide class of functions $K(x), S(x)$, (for single or multivariable $x$ ) using the Holonomic ansatz [Ze1], and implemented (for the single-variable case) in [AlZ], and for the multi-variable case in $[\mathrm{ApZ}]$, and much more efficiently in $[\mathrm{K}]$, there exists a linear recurrence equation with polynomial coefficients, that can be actually computed (always in theory, but also often in practice, unless the dimension is high). In other words we can find a positive integer $L$, the order of the recurrence, and polynomials $p_{0}(n), p_{1}(n), \ldots, p_{L}(n)$, such that

$$
p_{0}(n) I(n)+p_{1}(n) I(n+1)+\ldots+p_{L}(n) I(n+L)=0 \quad .
$$

If we are lucky (and all the cases in this paper fall into this case) the order $L$ is 2 . Furthermore, it would be evident in all the examples in this paper that $p_{0}(n), p_{1}(n), p_{2}(n)$ can be taken to have integer coefficients.

Another 'miracle' that happens in all the examples in this paper is that $I(0)$ and $I(1)$ are rationallyrelated, i.e. there exist integers $c_{0}, c_{1}, c_{2}$ such that

$$
c_{0} I(0)+c_{1} I(1)=c_{2} \quad,
$$


that our computers can easily find.

It then follows, by induction, that one can write

$$
I(n)=b_{n} C-a_{n},
$$

for some sequences of rational numbers $\left\{a_{n}\right\}$ and $\left\{b_{n}\right\}$ that both satisfy the same recurrence as $I(n)$.

Either using trivial bounds on the integral, or using the so-called Poincaré lemma (see, e.g. [vdP], $[\mathrm{ZeZu1}],[\mathrm{ZeZu} 2])$ it turns out that

$$
a_{n}=\Omega\left(\alpha^{n}\right) \quad, \quad b_{n}=\Omega\left(\alpha^{n}\right),
$$

for some constant $\alpha>1$, and

$$
|I(n)|=\Omega\left(\frac{1}{\beta^{n}}\right)
$$

for some constant $\beta>1$.

[Please note that we use $\Omega$ in a looser-than-usual sense, for us $x(n)=\Omega\left(\alpha^{n}\right)$ means that $\lim _{n \rightarrow \infty} \frac{\log x(n)}{n}=$ $\alpha$.

In the tweaks of Beukers' integrals for $\zeta(2)$ and $\zeta(3)$ coming up later, $\alpha$ and $\beta$ are equal, but in the tweaks of the Alladi-Robinson integrals, $\alpha$ is usually different than $\beta$.

It follows that

$$
\left|C-\frac{a_{n}}{b_{n}}\right|=\Omega\left(\frac{1}{(\alpha \beta)^{n}}\right)
$$

Note that $a_{n}$, and $b_{n}$ are, usually, not integers, but rather rational numbers (In the original Beukers/Apéry cases, the $b_{n}$ were integers, but the $a_{n}$ were not, in the more general cases in this article, usually neither of them are integers).

It so happens, in all the cases that we discovered, that there exists another sequence of rational numbers $E(n)$ such that

$$
a_{n}^{\prime}:=a_{n} E(n) \quad, \quad b_{n}^{\prime}:=b_{n} E(n) \quad,
$$

are always integers, and, of course $\operatorname{gcd}\left(a_{n}^{\prime}, b_{n}^{\prime}\right)=1$. We call $E(n)$ the integer-ating factor.

In some cases we were able to conjecture $E(n)$ exactly, in terms of products of primes satisfying certain conditions (see below), but in other cases we can only conjecture that such an explicitlydescribable sequence exists.

In either case there exists a real number, that sometimes can be described exactly, and other times only estimated, let's call it $\nu$, such that

$$
\lim _{n \rightarrow \infty} \frac{\log E(n)}{n}=\nu
$$


or, in our notation, $E(n)=\Omega\left(e^{n \nu}\right)$.

Since we have

$$
\left|C-\frac{a_{n}^{\prime}}{b_{n}^{\prime}}\right|=\Omega\left(\frac{1}{(\alpha \beta)^{n}}\right),
$$

where $b_{n}^{\prime}=\Omega\left(e^{\nu n} \alpha^{n}\right)$. We need a positive $\delta$ such that

$$
\left(e^{\nu n} \alpha^{n}\right)^{1+\delta}=(\alpha \beta)^{n} .
$$

Taking $\log$ (and dividing by $n$ ) we have

$$
(\nu+\log \alpha)(1+\delta)=\log \alpha+\log \beta \quad,
$$

giving

$$
\delta=\frac{\log \beta-\nu}{\log \alpha+\nu} .
$$

If we are lucky, and $\log \beta>\nu$, then we have $\delta>0$, and an irrationality proof!, Yea! We also, at the same time, determined an irrationality measure (see $[\mathrm{vdP}]$ )

$$
1+\frac{1}{\delta}=\frac{\log \alpha+\log \beta}{\log \beta-\nu}
$$

If we are unlucky, and $\delta<0$, it is still an exponentially fast way to compute our constant $C$ to any desired accuracy.

Summarizing: For each specific constant defined by a definite integral, we need to exhibit

- A second-oder recurrence equation for the numerator and denominator sequence $a_{n}$ and $b_{n}$ that feature in $I(n)=b_{n} C-a_{n}$.

- The initial conditions $a_{0}, a_{1}, b_{0}, b_{1}$ enabling a very fast computation of many terms of $a_{n}, b_{n}$.

- The constants $\alpha$ and $\beta$

- Exhibit a conjectured integer-ating factor $E(n)$, or else conjecture that one exists, and find, or estimate (respectively), $\nu:=\lim _{n \rightarrow \infty} \frac{\log E(n)}{n}$.

- Verify that $\beta>e^{\nu}$ and get (potentially) famous.

\section{The Three Classical Cases}

$\log 2([\mathrm{AR}])$

$$
\begin{aligned}
& C=\int_{0}^{1} \frac{1}{1+x} d x=\log 2 \\
& I(n)=\int_{0}^{1} \frac{(x(1-x))^{n}}{(1+x)^{n+1}} d x
\end{aligned}
$$


Recurrence:

$$
\begin{gathered}
(n+1) X(n)+(-6 n-9) X(n+1)+(n+2) X(n+2)=0 . \\
\alpha=\beta=3+2 \sqrt{2} .
\end{gathered}
$$

Initial conditions

$$
a_{0}=0, a_{1}=2 \quad ; \quad b_{0}=1, b_{1}=3 .
$$

Integer-ating factor $E(n)=l c m(1 \ldots n), \nu=1$.

$$
\delta=\frac{\log \beta-\nu}{\log \alpha+\nu}=\frac{\log \beta-1}{\log \alpha+1}=\frac{\log (3+2 \sqrt{2})-1}{\log (3+2 \sqrt{2})+1}=0.276082871862633587
$$

Implied irrationality measure: $1+1 / \delta=4.622100832454231334 \ldots$

$\zeta(2)([\mathrm{B}])$

$$
\begin{gathered}
C=\int_{0}^{1} \int_{0}^{1} \frac{1}{1-x y} d x d y=\zeta(2) . \\
I(n)=\int_{0}^{1} \int_{0}^{1} \frac{(x(1-x) y(1-y))^{n}}{(1-x y)^{n+1}} d x d y .
\end{gathered}
$$

Recurrence:

$$
\begin{gathered}
-(1+n)^{2} X(n)+\left(11 n^{2}+33 n+25\right) X(n+1)+(2+n)^{2} X(n+2)=0 . \\
\alpha=\beta=\frac{11}{2}+\frac{5 \sqrt{5}}{2} .
\end{gathered}
$$

Initial conditions

$$
a_{0}=0, a_{1}=-5 \quad ; \quad b_{0}=1, b_{1}=-3 .
$$

Integer-ating factor $E(n)=\operatorname{lcm}(1 \ldots n)^{2}, \nu=2$.

$$
\delta=\frac{\log \beta-\nu}{\log \alpha+\nu}=\frac{\log \beta-2}{\log \alpha+2}=\frac{\log (11 / 2+5 \sqrt{5} / 2)-2}{\log (11 / 2+5 \sqrt{5} / 2)+2}=0.09215925473323 \ldots
$$

Implied irrationality measure: $1+1 / \delta=11.8507821910523426959528 \ldots \ldots$ 
$\zeta(3)([\mathrm{B}])$

$$
\begin{gathered}
C=\int_{0}^{1} \int_{0}^{1} \int_{0}^{1} \frac{1}{1-z+x y z} d x d y d z=\zeta(3) \\
I(n)=\int_{0}^{1} \int_{0}^{1} \int_{0}^{1} \frac{(x(1-x) y(1-y) z(1-z))^{n}}{(1-z+x y z)^{n+1}} d x d y d z .
\end{gathered}
$$

Recurrence:

$$
\begin{gathered}
(1+n)^{3} X(n)-(2 n+3)\left(17 n^{2}+51 n+39\right) X(n+1)+(n+2)^{3} X(n+2)=0 . \\
\alpha=\beta=17+12 \sqrt{2} .
\end{gathered}
$$

Initial conditions

$$
a_{0}=0, a_{1}=12 \quad ; \quad b_{0}=1, b_{1}=5 .
$$

Integer-ating factor $E(n)=l c m(1 \ldots n)^{3}, \nu=3$.

$$
\delta=\frac{\log \beta-\nu}{\log \alpha+\nu}=\frac{\log \beta-3}{\log \alpha+3}=\frac{\log (17+12 \sqrt{2})-3}{\log (17+12 \sqrt{2})+3}=0.080529431189061685186 \ldots
$$

Implied irrationality measure: $1+1 / \delta=13.41782023335376578458 \ldots$

\section{Accompanying Maple packages}

This article is accompanied by three Maple packages, GenBeukersLog.txt, GenBeukersZeta2.txt, GenBeukersZeta3.txt all freely available from the front of this masterpiece

https://sites.math.rutgers.edu/〜zeilberg/mamarim/mamarimhtml/beukers.html, ,

where one can find ample sample input and output files, that readers are welcome to extend.

\section{Zudilin's Tweak of the Beukers $\zeta(2)$ integral to get the Catalan constant}

The inspiration for our tweaks came from Wadim Zudilin's brilliant discovery [Zu1] that the famous Catalan constant, that may be defined by the innocent-looking alternating series of the reciprocals of the odd perfect-squares

$$
C:=1-\frac{1}{3^{2}}+\frac{1}{5^{2}}-\frac{1}{7^{2}}+\ldots=\sum_{n=0}^{\infty} \frac{(-1)^{n}}{(2 n+1)^{2}}
$$


can be written as the double integral

$$
\frac{1}{8} \int_{0}^{1} \int_{0}^{1} \frac{x^{-\frac{1}{2}}(1-y)^{-\frac{1}{2}}}{1-x y} d x d y
$$

This lead him to consider the sequence of Beukers-type double-integrals

$$
I(n)=\int_{0}^{1} \int_{0}^{1} \frac{x^{-\frac{1}{2}}(1-y)^{-\frac{1}{2}}}{1-x y} \cdot\left(\frac{x(1-x) y(1-y)}{1-x y}\right)^{n} d x d y
$$

Using the Zeilberger algorithm, Zudilin derived a three term recurrence for $I(n)$ leading to good diophantine approximations to the Catalan constant, alas not good enough to prove irrationality. This was elaborated and extended by Yu. V. Nesterenko [N]. See also [Zu2].

Using the multivariable Almkvist-Zeilberger algorithm we can derive the recurrence much faster. Using Koutschan's package [K], it is yet faster.

\section{Our Tweaks}

Inspired by Zudilin's Beukers-like integral for the Catalan constant, we decided to use our efficient tools for quickly manufacturing recurrences.

We systematically investigated the following families.

\section{Generalizing the Alladi-Robinson-Like Integral for $\log 2$}

Alladi and Robinson $[\mathrm{AR}]$ gave a Beukers-style new proof of the irrationality of $\log 2$ using the elementary fact that

$$
\log 2=\int_{0}^{1} \frac{1}{1+x} d x
$$

and more generally,

$$
\frac{1}{c} \log (1+c)=\int_{0}^{1} \frac{1}{1+c x} d x
$$

They used the sequence of integrals

$$
I(n):=\int_{0}^{1} \frac{1}{1+c x}\left(\frac{x(1-x)}{1+c x}\right)^{n} d x
$$

and proved that for a wide range of choices of rational $c$, this leads to irrationality proofs and irrationality measures (see also [ZeZu1]).

Our generalized version is the three-parameter family of constants

$$
I_{1}(a, b, c):=\frac{1}{B(1+a, 1+b)} \int_{0}^{1} \frac{x^{a}(1-x)^{b}}{1+c x} d x
$$


that is easily seen to equal ${ }_{2} F_{1}(1, a+1 ; a+b+2 ;-c)$.

We use the sequence of integrals

$$
I_{1}(a, b, c)(n):=\frac{1}{B(1+a, 1+b)} \int_{0}^{1} \frac{x^{a}(1-x)^{b}}{1+c x} \cdot\left(\frac{x(1-x)}{1+c x}\right)^{n} d x .
$$

Using the (original!) Almkvist-Zeilberger algorithm [AlZ], implemented in the Maple package

https://sites.math.rutgers.edu/ zeilberg/tokhniot/EKHAD.txt ,

we immediately get a second-order recurrence that can be gotten by typing 'OpeL ( $\mathrm{a}, \mathrm{b}, \mathrm{c}, \mathrm{n}, \mathrm{N})$;' in the Maple package

https://sites.math.rutgers.edu/ ${ }^{\sim}$ zeilberg/tokhniot/GenBeukersLog.txt .

This enabled us to conduct a systematic search, and we found many cases of ${ }_{2} F_{1}$ evaluations that lead to irrationality proofs, i.e. for which the $\delta$ mentioned above is positive. Many of them turned out to be (conjecturally) expressible in terms of algebraic numbers and/or logarithms of rational numbers, hence proving them irrational is not that exciting, but we have quite a few not-yet-identified (and inequivalent) cases. See the output file

https://sites.math.rutgers.edu/ zeilberg/tokhniot/oGenBeukersLog1.txt ,

for many examples. Whenever Maple was able to (conjecturally) identify the constants explicitly, it is mentioned. If nothing is mentioned then these are potentially explicit constants, expressible as a hypergeometric series ${ }_{2} F_{1}$, for which this would be the first irrationality proof, once the details are filled-in.

We also considered the four-parameter family of constants

$$
I_{1}^{\prime}(a, b, c, d):=\frac{\int_{0}^{1} \frac{x^{a}(1-x)^{b}}{(1+c x)^{d+1}} d x}{\int_{0}^{1} \frac{x^{a}(1-x)^{b}}{(1+c x)^{d}} d x},
$$

and, using the more general recurrence, also obtained using the Almkvist-Zeilberger algorithm (to see it type 'OpeLg ( $a, b, c, d, n, S n)$;' in GenBeukersLog. txt), found many candidates for irrationality proofs that Maple was unable to identify. See the output file

https://sites.math.rutgers.edu/ ${ }^{\sim}$ zeilberg/tokhniot/oGenBeukersLog2.txt .

\section{Generalizing the Beukers Integral for $\zeta(2)$}

Define

$$
\begin{gathered}
I_{2}\left(a_{1}, a_{2}, b_{1}, b_{2}\right)(n)=\frac{1}{B\left(1-a_{1}, 1-a_{2}\right) B\left(1-b_{1}, 1-b_{2}\right)} \\
\int_{0}^{1} \int_{0}^{1} \frac{x^{-a_{1}}(1-x)^{-a_{2}} y^{-b_{1}}(1-y)^{-b_{2}}}{1-x y} \cdot\left(\frac{x(1-x) y(1-y)}{1-x y}\right)^{n} d x d y
\end{gathered}
$$


that happens to satisfy a linear-recurrence equation of second order, yielding Diophantine approximations to the constant $I_{2}\left(a_{1}, a_{2}, b_{1}, b_{2}\right)(0)$, let's call it $C_{2}\left(a_{1}, a_{2}, b_{1}, b_{2}\right)$

$$
C_{2}\left(a_{1}, a_{2}, b_{1}, b_{2}\right)=\frac{1}{B\left(1-a_{1}, 1-a_{2}\right) B\left(1-b_{1}, 1-b_{2}\right)} \cdot \int_{0}^{1} \int_{0}^{1} \frac{x^{-a_{1}}(1-x)^{-a_{2}} y^{-b_{1}}(1-y)^{-b 2}}{1-x y} d x d y \quad .
$$

It is readily seen that

$$
C_{2}\left(a_{1}, a_{2}, b_{1}, b_{2}\right)={ }_{3} F_{2}\left(\begin{array}{c}
1,1-a_{1},-b_{1}+1 \\
2-a_{1}-a_{2}, 2-b_{1}-b_{2}
\end{array} ; 1\right) .
$$

Most choices of random $a_{1}, a_{2}, b_{1}, b_{2}$ yield disappointing, negative $\delta$ 's, just like $C_{2}\left(\frac{1}{2}, 0,0, \frac{1}{2}\right)$ (alias 8 times the Catalan constant), but a systematic search yielded several hundred candidates that produce positive $\delta$ 's and hence would produce irrationality proofs. Alas, many of them were conjecturally equivalent to each other via a fractional-linear transformation with integer coefficients, $C \rightarrow \frac{a+b C}{c+d C}$, with $a, b, c, d$ integers, hence the facts that they are irrational are equivalent. Nevertheless we found quite a few that are (conjecturally) not equivalent to each other. Modulo filling-in some details, they lead to irrationality proofs. Amongst them some were (conjecturally) identified by Maple to be either algebraic, or logarithms of rational numbers, for which irrationality proofs exist for thousands of years (in case of $\sqrt{2}$ and $\sqrt{3}$ etc.), or a few hundred years (in case of $\log 2$, etc.).

But some of them Maple was unable to identify, so potentially our (sketches) of proofs would be the first irrationality proofs.

\section{Beukers $\zeta(2)$ Tweaks That produced Irrationality Proofs with Identified Constants}

\section{Denominator 2}

We first searched for $C_{2}\left(a_{1}, a_{2}, b_{1}, b_{2}\right)$ where the parameters $a_{1}, a_{2}, b_{1}, b_{2}$ have denominator 2 , there were quite a few of them, but they were all conjecturally equivalent to each other. Here is one of them:

- $C_{2}\left(0,0, \frac{1}{2}, 0\right)={ }_{3} F_{2}(1,1,1 / 2 ; 2,3 / 2 ; 1)$, alias $2 \log 2$.

\section{Denominator 3}

There were also quite a few where the parameters $a_{1}, a_{2}, b_{1}, b_{2}$ have denominator 3 , but again they were all equivalent to each other, featuring $\pi \sqrt{3}$. Here is one of them.

- $C_{2}\left(0,0, \frac{1}{3},-\frac{2}{3}\right)={ }_{3} F_{2}(1,1,2 / 3 ; 2,7 / 3 ; 1)$, alias (conjecturally) $-6+4 \pi \sqrt{3} / 3$.

\section{Denominator 4}

There were also quite a few where the parameters $a_{1}, a_{2}, b_{1}, b_{2}$ have denominator 4 , but again they were all equivalent to each other, featuring $\sqrt{2}$, yielding a new proof of the irrationality of $\sqrt{2}$ (for what it is worth). Here is one of them. 
- $C_{2}\left(-\frac{3}{4},-\frac{3}{4},-\frac{1}{4},-\frac{3}{4}\right)={ }_{3} F_{2}(1,7 / 4,5 / 4 ; 7 / 2,3 ; 1)$, alias (conjecturally) $-240+\frac{512}{3} \sqrt{2}$.

\section{Denominator 5}

There were also quite a few where the parameters $a_{1}, a_{2}, b_{1}, b_{2}$ have denominator 5 , but again they were all equivalent to each other, featuring $\sqrt{5}$, yielding a new proof of the irrationality of $\sqrt{5}$ (for what it is worth). Here is one of them.

- $C_{2}\left(-\frac{4}{5},-\frac{4}{5},-\frac{2}{5},-\frac{3}{5}\right)={ }_{3} F_{2}(1,9 / 5,7 / 5 ; 18 / 5,3 ; 1)$, alias (conjecturally) $-\frac{845}{2}+\frac{2275}{12} \sqrt{5}$

\section{Denominator 6 with identified constants}

We found two equivalence classes where the parameters $a_{1}, a_{2}, b_{1}, b_{2}$ have denominator 6 , for which the constants were identified. Here are one from each class.

- $C_{2}(-5 / 6,-5 / 6,-1 / 2,-1 / 2)={ }_{3} F_{2}(1,11 / 6,3 / 2 ; 11 / 3,3 ; 1)$, alias (conjecturally) $-\frac{1344}{5}+\frac{16384 \sqrt{3}}{105}$

- $C_{2}(-5 / 6,-5 / 6,-1 / 3,-2 / 3)={ }_{3} F_{2}(1,11 / 6,4 / 3 ; 11 / 3,3 ; 1)$, alias (conjecturally) $\frac{9722^{2 / 3}}{5}-\frac{1536}{5}$

\section{denominator 7 with identified constants}

We found two cases where the parameters $a_{1}, a_{2}, b_{1}, b_{2}$ have denominator 7 , for which the constants were identified.

- $C_{2}(-6 / 7,-6 / 7,-4 / 7,-3 / 7)={ }_{3} F_{2}(1,13 / 7,11 / 7 ; 26 / 7,3 ; 1)$, alias (conjecturally) the positive root of $13824 x^{3}-2757888 x^{2}-10737789048 x+16108505539=0$.

- $C_{2}(-6 / 7,-1 / 7,4 / 7,2 / 7)={ }_{3} F_{2}(1,13 / 7,3 / 7 ; 3,8 / 7 ; 1)$, alias (conjecturally) the positive root of $2299968 x^{3}+7074144 x^{2}-11234916 x-12663217=0$

\section{Beukers $\zeta(2)$ Tweaks That produced Irrationality Proofs with Not-Yet-Identified Con- stants (and Hence Candidates for First Irrationality Proofs)}

For the following constants, Maple was unable to identify, and we have potentially the first irrationality proofs of these constants.

\section{Denominator 6 with not yet identified constants}

We found two cases (up to equivalence):

- $C_{2}(0,-1 / 2,1 / 6,-1 / 2)={ }_{3} F_{2}(1,1,5 / 6 ; 5 / 2,7 / 3 ; 1)$

While Maple was unable to identify this constant, Mathematica came up with $-24-\frac{81 \sqrt{\pi} \Gamma(7 / 3)}{\Gamma(-1 / 6)}$.

- $C_{2}(-2 / 3,-1 / 2,1 / 2,-1 / 2)={ }_{3} F_{2}(1,5 / 3,1 / 2 ; 19 / 6,2 ; 1)$

While Maple was unable to identify this constant, Mathematica came up with $\frac{13}{2}-\frac{6 \Gamma(19 / 6)}{\sqrt{\pi} \Gamma(8 / 3)}$. 


\section{Denominator 7 with not yet identified constants}

We found six cases (up to equivalence):

- $C_{2}(-6 / 7,-6 / 7,-4 / 7,-5 / 7)={ }_{3} F_{2}(1,13 / 7,11 / 7 ; 26 / 7,23 / 7 ; 1)$

- $C_{2}(-6 / 7,-5 / 7,-3 / 7,-5 / 7)={ }_{3} F_{2}(1,13 / 7,10 / 7 ; 25 / 7,22 / 7 ; 1)$

- $C_{2}(-6 / 7,-5 / 7,-2 / 7,-1 / 7)={ }_{3} F_{2}(1,13 / 7,9 / 7 ; 25 / 7,17 / 7 ; 1)$

- $C_{2}(-6 / 7,-4 / 7,-1 / 7,-1 / 7)={ }_{3} F_{2}(1,13 / 7,8 / 7 ; 24 / 7,16 / 7 ; 1)$

- $C_{2}(-6 / 7,-3 / 7,-5 / 7,-3 / 7)={ }_{3} F_{2}(1,13 / 7,12 / 7 ; 23 / 7,22 / 7 ; 1)$

- $C_{2}(-5 / 7,-3 / 7,-4 / 7,-2 / 7)={ }_{3} F_{2}(1,12 / 7,11 / 7 ; 22 / 7,20 / 7 ; 1)$

For each of them, to get the corresponding theorem and proof, use procedure Theoremz2 in the Maple pacgage GenBeukersZeta2.txt.

To get a statement and full proof (modulo a divisibility lemma) type, in GenBeukersZeta2.txt TheoremZ2 (a1, a2 , b1, b2, K, 0):

with $\mathrm{K}$ at least 2000. For example, for the last constant in the above list ${ }_{3} F_{2}(1,12 / 7,11 / 7 ; 22 / 7,20 / 7 ; 1)$, type

TheoremZ2 $(-5 / 7,-3 / 7,-4 / 7,-2 / 7,3000,0)$ :

For more details (the recurrences, the estimated irrationality measures, the initial conditions) see the output file

https://sites.math.rutgers.edu/ zeilberg/tokhniot/oGenBeukersZeta2g.txt .

\section{Generalizing the Beukers Integral for $\zeta(3)$}

The natural extension would be the six-parameter family (but now we make the exponents positive)

$$
\begin{gathered}
\frac{1}{B\left(1+a_{1}, 1+a_{2}\right) B\left(1+b_{1}, 1+b_{2}\right) B\left(1+c_{1}, 1+c_{2}\right)} \\
\int_{0}^{1} \int_{0}^{1} \int_{0}^{1} \frac{x^{a_{1}}(1-x)^{a_{2}} y^{b_{1}}(1-y)^{b_{2}} z^{c_{1}}(1-z)^{c_{2}}}{1-z+x y z} \cdot\left(\frac{x(1-x) y(1-y) z(1-z)}{1-z+x y z}\right)^{n} d x d y d z
\end{gathered}
$$

However, for arbitrary $a_{1}, a_{2}, b_{1}, b_{2}, c_{1}, c_{2}$ the recurrence is third order. (Wadim Zudilin pointed out that this may be related to the work of Rhin and Viola in [RV]).

Also, empirically, we did not find many promising cases. Instead, let's define

$$
J_{3}\left(a_{1}, a_{2}, b_{1}, b_{2}, c_{1}, c_{2} ; e\right)(n)
$$




$$
\int_{0}^{1} \int_{0}^{1} \int_{0}^{1} \frac{x^{a_{1}}(1-x)^{a_{2}} y^{b_{1}}(1-y)^{b_{2}} z^{c_{1}}(1-z)^{c_{2}}}{(1-z+x y z)^{e}} \cdot\left(\frac{x(1-x) y(1-y) z(1-z)}{1-z+x y z}\right)^{n} d x d y d z
$$

and

$$
I_{3}\left(a_{1}, a_{2}, b_{1}, b_{2}, c_{1}, c_{2} ; e\right)(n):=\frac{J_{3}\left(a_{1}, a_{2}, b_{1}, b_{2}, c_{1}, c_{2} ; e+1\right)(n)}{J_{3}\left(a_{1}, a_{2}, b_{1}, b_{2}, c_{1}, c_{2} ; e\right)(0)}
$$

The family of constants that we hope to prove irrationality is the five-parameter:

$$
\begin{gathered}
I_{3}\left(a_{1}, a_{2}, b_{1}, b_{2}, c_{1}, c_{2} ; e\right)(0) \\
=\frac{\int_{0}^{1} \int_{0}^{1} \int_{0}^{1} \frac{x^{a_{1}}(1-x)^{a_{2}} y^{b_{1}}(1-y)^{b_{2}} z^{c_{1}}(1-z)^{c_{2}}}{(1-z+x y z)^{e^{+1}}} d x d y d z}{\int_{0}^{1} \int_{0}^{1} \int_{0}^{1} \frac{x^{a_{1}}(1-x)^{a_{2}} y^{b_{1}}(1-y)^{b_{2}} z^{c_{1}}(1-z)^{c_{2}}}{(1-z+x y z)^{e}} d x d y d z} .
\end{gathered}
$$

Of course, for this more general, 7-parameter, family, there is no second-order recurrence, but rather a third-order one. But to our delight, we found a five-parameter family, let's call it

$$
K(a, b, c, d, e)(n):=I_{3}(b, c, e, a, a, c, d)(n) \quad .
$$

Spelled-out, our five-parameter family of constants is

$$
\begin{gathered}
K(a, b, c, d, e)(0)= \\
\frac{\int_{0}^{1} \int_{0}^{1} \int_{0}^{1} \frac{x^{b}(1-x)^{c} y^{e}(1-y)^{a} z^{a}(1-z)^{c}}{(1-z+x y z)^{d+1}} d x d y d z}{\int_{0}^{1} \int_{0}^{1} \int_{0}^{1} \frac{x^{b}(1-x)^{c} y^{e}(1-y)^{a} z^{a}(1-z)^{c}}{(1-z+x y z)^{d}} d x d y d z} .
\end{gathered}
$$

Now we found (see the section on finding recurrences below) a general second-order recurrence, that is too complicated to display here in full generality, but can be seen by typing

$\operatorname{OPEZ3}(\mathrm{a}, \mathrm{b}, \mathrm{c}, \mathrm{d}, \mathrm{e}, \mathrm{n}, \mathrm{Sn})$;

In the Maple package GenBeukersZeta3.txt. This enabled us, for each specific, numeric specialization of the parameters $a, b, c, d, e$ to quickly find the relevant recurrence, and systematically search for those that give positive $\delta$. Once again, many of them turned out to be (conjecturally) equivalent to each other.

\section{Denominator 2:}

We only found one class, up to equivalence, all related to $\log 2$. One of them is

$$
K(0,0,0,1 / 2,1 / 2)=I_{3}(0,0,1 / 2,0,0,0,1 / 2) \quad,
$$

that is not that exciting since it is (conjecturally) equal to $-\frac{2-4 \log (2)}{3-4 \log (2)}$.

For details, type TheoremZ3 $(0,0,0,1 / 2,1 / 2,3000,0)$; in GenBeukersZeta3.txt . 


\section{Denominator 3:}

We found three inequivalent classes, none of them Maple was able to identify.

$$
K(0,0,0,1 / 3,2 / 3)=I_{3}(0,0,2 / 3,0,0,0,1 / 3) \quad,
$$

for details, type TheoremZ3 $(0,0,0,1 / 3,2 / 3,3000,0)$; in GenBeukersZeta3.txt.

$$
K(0,0,0,2 / 3,1 / 3)=I_{3}(0,0,1 / 3,0,0,0,2 / 3) \quad,
$$

for details, type TheoremZ3 $(0,0,0,2 / 3,1 / 3,3000,0)$; in GenBeukersZeta3.txt.

$$
K(0,1 / 3,2 / 3,1 / 3,2 / 3)=I_{3}(0,0,1 / 3,0,0,0,2 / 3) \quad,
$$

for details, type TheoremZ3 $(0,1 / 3,2 / 3,1 / 3,2 / 3,3000,0)$; in GenBeukersZeta3.txt,

These three constants are candidates for 'first-ever-irrationality proof'.

Denominator 4: We only found one family, all expressible in terms of log 2. Here is one of them.

For example

$$
K(0,1 / 2,0,1 / 4,3 / 4)=I_{3}(1 / 2,0,3 / 4,0,0,0,1 / 4) \quad,
$$

that, conjecturally equals $-\frac{-30+45 \log (2)}{-11+15 \log (2)}$.

For details, type TheoremZ3 $(0,1 / 2,0,1 / 4,3 / 4,3000,0)$; in GenBeukersZeta3.txt.

Denominator 5: We only found one family, up to equivalence, but Maple was unable to identify the constant. So it is potentially the first irrationality proof of that constant

$$
K(0,1 / 5,0,3 / 5,2 / 5)=I_{3}(1 / 5,0,2 / 5,0,0,0,3 / 5)
$$

For details, type TheoremZ3 $(0,1 / 5,0,3 / 5,2 / 5,3000,0)$; in GenBeukersZeta3.txt.

Denominator 6: We found three families, up to equivalence, none of which Maple was able to identify. Once again, these are candidates for first-ever irrationality proofs for these constants.

$$
K(0,1 / 2,1 / 2,1 / 3,1 / 6)=I_{3}(1 / 2,1 / 2,1 / 6,0,0,1 / 2,1 / 3) \quad .
$$

For details, type TheoremZ3 $(0,1 / 2,1 / 2,1 / 3,1 / 6,3000,0)$; in GenBeukersZeta3.txt.

$$
K(0,1 / 2,1 / 2,1 / 6,1 / 3)=I_{3}(1 / 2,1 / 2,1 / 3,0,0,1 / 2,1 / 6) \quad .
$$

For details, type TheoremZ3 $(0,1 / 2,1 / 2,1 / 6,1 / 3,3000,0)$; in GenBeukersZeta3.txt. 


$$
K(1 / 3,0,2 / 3,1 / 2,5 / 6)=I_{3}(0,2 / 3,5 / 6,1 / 3,1 / 3,2 / 3,1 / 2) \quad .
$$

For details, type TheoremZ3 $(1 / 3,0,2 / 3,1 / 2,5 / 6,3000,0)$; in GenBeukersZeta3.txt.

Denominator 7: We found five families, up to equivalence, none of which Maple was able to identify. Once again, these are candidates for first-ever irrationality proofs for these constants.

$$
K(1 / 7,0,2 / 7,3 / 7,4 / 7)=I_{3}(0,2 / 7,4 / 7,1 / 7,1 / 7,2 / 7,3 / 7) \quad .
$$

For details, type TheoremZ3 $(1 / 7,0,2 / 7,3 / 7,4 / 7,3000,0)$; in GenBeukersZeta3.txt.

$$
K(1 / 7,0,2 / 7,5 / 7,3 / 7)=I_{3}(0,2 / 7,3 / 7,1 / 7,1 / 7,2 / 7,5 / 7) \quad .
$$

For details, type TheoremZ3 $(1 / 7,0,2 / 7,5 / 7,3 / 7,3000,0)$; in GenBeukersZeta3.txt.

$$
K(1 / 7,0,3 / 7,4 / 7,5 / 7)=I_{3}(0,3 / 7,5 / 7,1 / 7,1 / 7,3 / 7,4 / 7) \quad .
$$

For details, type TheoremZ3 $(1 / 7,0,3 / 7,4 / 7,5 / 7,3000,0)$; in GenBeukersZeta3.txt.

$$
K(1 / 7,0,4 / 7,2 / 7,5 / 7)=I_{3}(0,4 / 7,5 / 7,1 / 7,1 / 7,4 / 7,2 / 7) \quad .
$$

For details, type TheoremZ3 $(1 / 7,0,4 / 7,2 / 7,5 / 7,3000,0)$; in GenBeukersZeta3.txt.

$$
K(2 / 7,0,3 / 7,4 / 7,5 / 7)=I_{3}(0,3 / 7,5 / 7,2 / 7,2 / 7,3 / 7,4 / 7) \quad .
$$

For details, type TheoremZ3 $(2 / 7,0,3 / 7,4 / 7,5 / 7,3000,0)$; in GenBeukersZeta3.txt.

If you don't have Maple, you can look at the output file

https://sites.math.rutgers.edu/〜zeilberg/tokhniot/oGenBeukersZeta3All.txt ,

that gives detailed sketches of irrationality proofs of all the above constants, some with conjectured integer-ating factors.

\section{Guessing an INTEGER-ating factor}

In the original Beukers cases the integer-ating factor was easy to conjecture, and even to prove. For $\zeta(2)$ it was $l c m(1 \ldots n)^{2}$, and for $\zeta(3)$ it was $l c m(1 \ldots n)^{3}$. For the Alladi-Robinson case of $\log 2$ it was even simpler, $\operatorname{lcm}(1 \ldots n)$.

But in other cases it is much more complicated. A natural 'atomic' object is, given a modulo M, a subset $\mathrm{C}$ of $\{0, \ldots, M-1\}$, rational numbers $e_{1}, e_{2}$ between 0 and 1 , rational numbers $e_{3}, e_{4}$, the 
following quantity, for positive integers $n$

$$
P p\left(e_{1}, e_{2}, e_{3}, e_{4}, C, M ; n\right):=\prod_{p} p
$$

where $p$ ranges over all primes such that (let $\{a\}$ be the fractional part of $a$, i.e. $a-\lfloor a\rfloor$ )

- $e_{1}<\{n / p\}<e_{2}$

- $e_{3}<p / n<e_{4}$

- $p \bmod M \in C$

Using the prime number theorem, it follows (see e.g. [Zu2]) that

$$
\lim _{n \rightarrow \infty} \frac{\log P p\left(e_{1}, e_{2}, e_{3}, e_{4}, C, M ; n\right)}{n}
$$

can be evaluated exactly, in terms of the function $\Psi(x)=\frac{\Gamma^{\prime}(x)}{\Gamma(x)}$ (see procedure PpGlimit in the Maple packages) thereby giving an exact value for the quantity $\delta$ whose positivity implies irrationality.

Of course, one still needs to rigorously prove that the conjectured integer-ating factor is indeed correct.

\section{Looking under the hood: On Recurrence Equations}

For 'secrets from the kitchen' on how we found the second-order, four-parameter recurrence operator

OPEZ2 (a1 , a2 , b1 , b2 , n, N) in the Maple package GenBeukersZeta2.txt, that was the engine driving the $\zeta(2)$ tweaks, and more impressively, the five-parameter second-order recurrence operator OPEZ3 ( $a, b, c, d, e, n, N)$ in the Maple package GenBeukersZeta3.txt, that was the engine driving the $\zeta(3)$ tweaks, the reader is referred to the stand-alone appendix available from the following url:

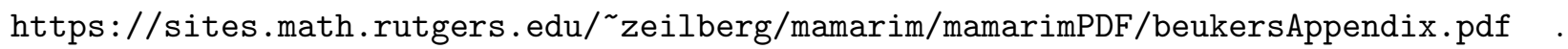

\section{Other Variations on Apéry's theme}

Other attempts to use Apéry's brilliant insight are [Ze2][Ze3][ZeZu1]. Recently Marc Chamberland and Armin Straub [CS] explored other fascinating aspects of the Apéry numbers, not related to irrationality.

\section{Conclusion and Future Work}

We believe that symbolic computational methods have great potential in irrationality proofs, in particular, and number theory in general. In this article we confined attention to approximating sequences that arise from second-order recurrences. The problem with higher order recurrences 
is that one gets linear combinations with rational coefficients of several constants, but if you can get two different such sequences coming from third-order recurrences, both featuring the same two constants, then the present method may be applicable. More generally if you have a $k$-th order recurrences, you need $k-1$ different integrals.

The general methodology of this article can be called Combinatorial Number Theory, but not in the usual sense, but rather as an analog of Combinatorial Chemistry, where one tries out many potential chemical compounds, most of them useless, but since computers are so fast, we can afford to generate lots of cases and pick the wheat from the chaff.

\section{Encore: Hypergeometric challenges}

As a tangent, we (or rather Maple) discovered many exact ${ }_{3} F_{2}(1)$ evaluations. Recall that the Zeilberger algorithm can prove hypergemoetric identities only if there is at least one free parameter. For a specific ${ }_{3} F_{2}\left(a_{1} a_{2} a_{3} ; b_{1} b_{2} ; 1\right)$, with numeric parameters, it is useless. Of course, it is sometimes possible to introduce such a parameter in order to conjecture a general identity, valid for 'infinitely' many $n$, and then specialize $n$ to a specific value, but this remains an art rather than a science. The output file

https://sites.math.rutgers.edu/ zeilberg/tokhniot/oGenBeukersZeta2f .txt

contains many such conjectured evaluations, (very possibly many of them are equivalent via a hypergeometric transformation rule) and we challenge Wadim Zudilin, the birthday boy, or anyone else, to prove them.

\section{References}

[AR] Krishna Alladi and Michael L. Robinson, Legendre polynomials and irrationality, J. Reine Angew. Math. 318 (1980), 137-155.

[AlZ] Gert Almkvist and Doron Zeilberger, The method of differentiating under the integral sign, J. Symbolic Computation 10, 571-591 (1990).

https://sites.math.rutgers.edu/〜zeilberg/mamarim/mamarimhtml/duis.html .

[ApaZ] Moa Apagodu and Doron Zeilberger, Multi-variable Zeilberger and Almkvist-Zeilberger algorithms and the sharpening of Wilf-Zeilberger Theory, Adv. Appl. Math. 37 (2006)(Special Regev issue), 139-152.

https://sites.math.rutgers.edu/ ${ }^{2}$ zeilberg/mamarim/mamarimhtml/multiZ.html .

[Ape] Roger Apéry, "Interpolation de fractions continues et irrationalité de certaine constantes" Bulletin de la section des sciences du C.T.H.S. \#3 p. 37-53, 1981.

[B] Frits Beukers, A note on the irrationality of $\zeta(2)$ and $\zeta(3)$, Bull. London Math. Soc. 11 (1979), 268-272. 
[CS] Marc Chamberland and Armin Straub, Apéry limits: Experiments and Proofs, arxiv:2001.034400v1, 6 Nov 2020.

https://arxiv.org/abs/2011.03400.

[H] Professor David Hilbert, Mathematical Problems [Lecture delivered before the International Congress of Mathematicians at Paris in 1900], translated by Dr. Mary Winston Newson, Bulletin of the American Mathematica Society 8 (1902), 437-479.

https : / www . ams . org/journals/bull/2000-37-04/S0273-0979-00-00881-8/S0273-0979-00-008818.pdf .

[K] Christoph Koutschan, Advanced applications of the holonomic systems approach, PhD thesis, Research Institute for Symbolic Computation (RISC), Johannes Kepler University, Linz, Austria, 2009.

http://www.koutschan.de/publ/Koutschan09/thesisKoutschan.pdf, http://www.risc.jku.at/research/combinat/software/HolonomicFunctions/ .

[N] Yu. V. Nesterenko, On Catalan's constant, Proceedings of the Steklov Institute of Mathematics 292 (2016), 153-170.

[vdP] Alf van der Poorten, A proof that Euler missed... Apéry's proof of the irrationality of $\zeta(3)$, Math. Intelligencer 1 (1979), 195-203.

[RV] Georges Rhin and Carlo Viola, The group structure of $\zeta(3)$, Acta Arithmetica, 97(2001), 269-293.

[Ze1] Doron Zeilberger, A Holonomic systems approach to special functions identities, J. of Computational and Applied Math. 32, 321-368 (1990).

https://sites.math.rutgers.edu/〜zeilberg/mamarim/mamarimhtml/holonomic.html .

[Ze2] Doron Zeilberger, Computerized deconstruction, Adv. Applied Math. 30 (2003), 633-654.

https://sites.math.rutgers.edu/ ${ }^{\sim}$ zeilberg/mamarim/mamarimhtml/derrida.html .

[Ze3] Doron Zeilberger, Searching for Apéry-style miracles [using, inter-alia, the amazing AlmkvistZeilberger algorithm], Personal Journal of Shalosh B. Ekhad and Doron Zeilberger, https://sites.math.rutgers.edu/ zeilberg/mamarim/mamarimhtml/apery.html .

[ZeZu1] Doron Zeilberger, and Wadim Zudilin, Automatic discovery of irrationality proofs and irrationality measures, International Journal of Number Theory, published on-line before print, volume and page tbd. Also to appear in a book dedicated to Bruce Berndt.

https://sites.math.rutgers.edu/〜zeilberg/mamarim/mamarimhtml/gat.html .

[ZeZu2] Doron Zeilberger, and Wadim Zudilin, The irrationality measure of Pi is at most 7.103205334137..., Moscow J. of Combinatorics and Number Theory 9 (2020), 407-419.

https://sites.math.rutgers.edu/ zeilberg/mamarim/mamarimhtml/pimeas.html . 
[Zu1] Wadim Zudilin, Apéry-like difference equations for Catalan's constant https://arxiv.org/abs/math/0201024.

[Zu2] Wadim Zudilin, Arithmetic of linear forms involving odd zeta values, J. Théorie Nombres Bordeaux 16 (2004), 251-291.

https://arxiv.org/abs/math/0206176.

Robert Dougherty-Bliss, Department of Mathematics, Rutgers University (New Brunswick), Hill Center-Busch Campus, 110 Frelinghuysen Rd., Piscataway, NJ 08854-8019, USA.

Email: Robert.w.Bliss at gmail dot com.

Christoph Koutschan, Johann Radon Institute of Computational and Applied Mathematics (RICAM), Austrian Academy of Sciences, Altenberger Strasse 69, A-4040 Linz, Austria Email: christoph.koutschan at ricam dot oeaw dot ac dot at .

Doron Zeilberger, Department of Mathematics, Rutgers University (New Brunswick), Hill CenterBusch Campus, 110 Frelinghuysen Rd., Piscataway, NJ 08854-8019, USA.

Email: DoronZeil at gmail dot com . 